
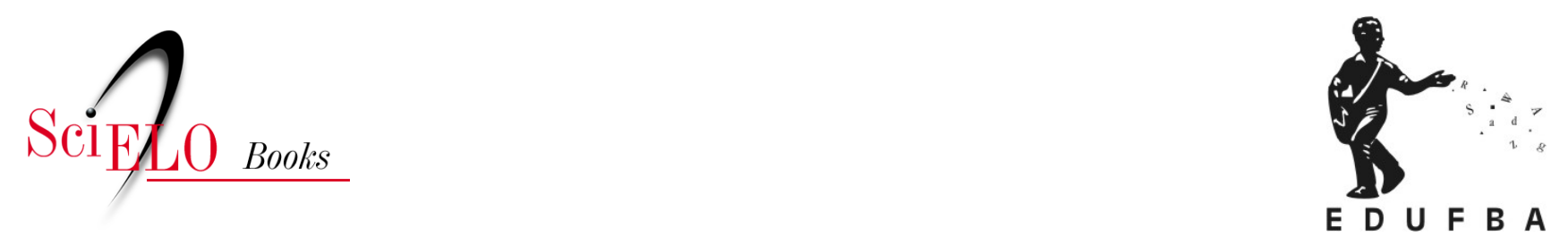

\title{
Produção científica sobre política de saúde no Brasil (1988-2014) contribuição ao debate sobre a Reforma Sanitária Brasileira
}

\author{
Jamilli Silva Santos \\ Carmen Fontes Teixeira
}

SANTOS, J.S., and TEIXEIRA, C.F. Produção científica sobre política de saúde no Brasil (1988-2014): contribuição ao debate sobre a Reforma Sanitária Brasileira. In: TEIXEIRA, C.F., comp. Observatório de análise política em saúde: abordagens, objetos e investigações [online]. Salvador: EDUFBA, 2016, pp. 41-72. ISBN 978-85-232-2021-1. https://doi.org/10.7476/9788523220211.0003.

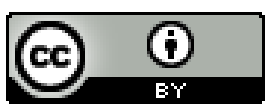

All the contents of this work, except where otherwise noted, is licensed under a Creative Commons Attribution 4.0 International license.

Todo o conteúdo deste trabalho, exceto quando houver ressalva, é publicado sob a licença Creative Commons Atribição $\underline{4.0}$. 


\section{PRODUÇÃO CIENTÍFICA SOBRE POLÍTICA DE SAÚDE NO BRASIL (1988-2014) contribuição ao debate sobre a Reforma Sanitária Brasileira}

\section{Introdução}

A Reforma Sanitária Brasileira (RSB) vem sendo estudada como um fenômeno sócio-histórico, conformado por múltiplos fatos produzidos por vários atores, cujas ações configuram um processo desencadeado na sociedade brasileira em meados dos anos $1970 \mathrm{e}$ ainda em curso. Enquanto proposta, foi resultante de um movimento da sociedade civil brasileira em defesa da democracia, dos direitos sociais e de um novo sistema de saúde. (ESCOREL, 1999; FLEURY, 1994; GERSCHMAN, 1985) Transformou-se em projeto a partir da $8^{a}$ Conferência Nacional de Saúde (BRASIL, 1986a) e desenvolveu-se como processo, especialmente com a instalação da Comissão Nacional de Reforma Sanitária. (BRASIL, 1986b) Desse modo, a RSB é simultaneamente uma proposta, um projeto, um movimento e um processo. (PAIM, 2008a) 
A ideia e a proposta da RSB foram esboçadas no contexto da nova república que anunciava a recuperação dos direitos políticos para os brasileiros, apontando para a construção de um estado ampliado, onde se fariam presentes maior leque de interesses de classes, frações de classes, segmentos sociais e grupos de interesses. Marcadas ainda pelas lutas contra o autoritarismo desde a fundação do Centro Brasileiro de Estudos em Saúde (A QUESTÃO..., 1979), tais ideia e proposta expressaram-se em um complexo projeto, formalizado na Constituição Federal de 1988 (BRASIL, 1988), o qual previa que as modificações necessárias ao setor saúde transcendiam os limites de uma reforma administrativa e financeira, exigindo uma reformulação mais profunda das concepções e práticas de saúde, incluindo a reforma do setor em seus aspectos políticos, organizacionais e técnico -operacionais (PAIM, 2008a), isto é, do seu arcabouço institucional, a partir da unificação, descentralização e democratização da gestão do sistema (NORONHA; LIMA; MACHADO, 2012), bem como da mudança do modelo de atenção à saúde, com ênfase na constru42 ção da integralidade do cuidado. (TEIXEIRA; VILASBÔAS, 2014)

Conformando-se, portanto, como um conjunto de práticas ideológicas, políticas e culturais que tomam a saúde como referente fundamental, o processo de RSB pode ser descrito e analisado a partir de um ciclo integrado pelos momentos que contemplam desde a elaboração da ideia, à conformação da proposta e sistematização de um projeto político incorporado parcialmente à agenda do Estado e traduzido em políticas e ações de saúde nos últimos 25 anos. (PAIM, 2008a; PAIM, 2013a; PAIM et al., 2011; TEIXEIRA; SOUZA; PAIM, 2014)

A compreensão desse processo perpassa pelo estudo das conjunturas conformadas desde a emergência da ideia até o momento atual. As análises e avaliações das políticas de saúde do governo Lula (BAHIA, 2010; MACHADO; BAPTISTA; LIMA, 2012; TEIXEIRA; PAIM, 2005) apontam continuidades e mudanças, embora sem questionarem ou aprofundarem a análise sobre o sentido do processo da RSB, assim como a sua possível permanência. Nem mesmo os 
obstáculos e as ameaças ao Sistema Único de Saúde (SUS), identificados na conjuntura atual por diversos estudos (BORGES et al., 2012; COSTA; BAHIA; SCHEFFER, 2013; NORONHA; SANTOS; PEREIRA, 2011; OCKÉ-REIS, 2012; PAIM, 2012; PAIM et al., 2011), estimularam, até o presente, a realização de pesquisas e uma apreciação crítica da RSB que alcançassem o final do período Lula e o governo Dilma Rousseff, especialmente diante das possíveis relações com projetos políticos em disputa na sociedade brasileira. (MAGNO, 2014)

Para além dessas discussões, não há consenso entre os estudiosos sobre a própria vitalidade da RSB. Hochman (2013) defende que a RSB findou-se como conjunto de ideias e práticas políticas contextualizadas em certo tempo de transição, resultado do seu próprio êxito na proposição de uma agenda política e criação de um sistema de saúde reconhecido mundialmente, bem como da conquista da democracia em 1985. Contrariamente, Paim (2013a) defende que a RSB enquanto processo não terminou, nem tem prazo de validade, o que pode ser evidenciado pela Agenda Estratégica para a Saúde (AGENDA..., 2011; UMA AGENDA..., 2011), pelas centenas de cidadãos formados pelo Centro Brasileiro de Estudos de Saúde (Cebes) nos últimos anos (SOPHIA, 2015) e pela movimentação de milhares de participantes dos congressos da Associação Brasileira de Saúde Coletiva (LIMA; SANTANA, 2006), bem como da participação cidadã em conselhos e conferências de saúde. (CÔRTES, 2009, 2010; LABRA; FIGUEIREDO, 2002; LOBATO; FLEURY, 2009)

A reflexão sobre essa polêmica gerou o interesse em se atualizar e aprofundar a revisão bibliográfica sobre política de saúde no período pós-constituinte, tomando-se como ponto de partida os estudos já realizados nessa linha. (LEVCOVITZ et al., 2003; PAIM; TEIXEIRA, 2006; TEIXEIRA; SOUZA; PAIM, 2014) Tomando como referencial o debate sobre a permanência ou esgotamento do processo de RSB, foram elaboradas as seguintes questões: 
a. quais as características da produção científica brasileira sobre política de saúde no período 1988-2014?

b. Quais as características da evolução temporal dessa produção?

c. Quais os principais temas e questóes abordadas nos estudos realizados neste período?

d. Até que ponto os estudos realizados expressam a ocorrência de mudanças na concepção dos pesquisadores em relação à $\mathrm{RSB}$ ?

Nessa perspectiva, o objetivo geral deste capítulo é apresentar os resultados de uma revisão sistemática da literatura mediante a aplicação de métodos explícitos e sistematizados de busca, apreciação crítica e síntese (SAMPAIO; MANCINI, 2007) de artigos selecionados na base Scientific Electronic Library Online (Scielo), em 2014, procedendo-se à sua classificação segundo temas e objetos de estudo, bem como a análise de conteúdo dos resumos da subárea "análise política em saúde”, nos quais se tratou de identificar até que ponto tais estudos reafirmam ou negam a permanência do processo de RSB 44 nas diversas conjunturas, desde 1988 até o momento atual. Com isso, tratamos de discutir a manutenção, mudança ou transformação de concepções que marcaram a emergência da RSB no âmbito da comunidade científica da área de Saúde Coletiva, indicando, indiretamente, os contornos das bases de sustentação política desse processo.

\section{Aspectos teórico-metodológicos}

Tomamos como ponto de partida o conceito proposto por Paim (2003), segundo o qual "política de saúde" é a resposta social (ação ou omissão) do Estado aos problemas e necessidades de saúde da população, contemplando, portanto, a intervenção sobre a produção, distribuição, gestão e regulação de bens e serviços que afetam a saúde, inclusive o ambiente. Desse modo, abrange as questões relativas ao poder em saúde (natureza, estrutura, relações, distribuição e lutas), bem como as que se referem ao estabelecimento de diretrizes, planos e programas de saúde. Ou seja, contempla tanto os planos de 
ação governamental (KINGDON, 2011) quanto a análise das relações de poder em saúde. (TESTA, 1995)

Enquanto disciplina acadêmica, Política de Saúde inclui estudos sobre o papel do Estado, as relações Estado-Sociedade, movimentos sociais em saúde, as relações entre políticas de saúde e políticas econômicas e sociais (LEVCOVITZ et al., 2003; PAIM, 2003) e outros aspectos relativos à análise dos processos políticos que ocorrem em diversos espaços sociais. Também abarca estudos que tratam da formulação e implementação de políticas específicas no âmbito governamental, abordando, por exemplo, a conformação da agenda política em saúde, a elaboração de propostas de intervenção sobre problemas de grupos populacionais específicos bem como a gestão, implantação e avaliação de planos, programas e projetos.

Neste trabalho, o mapeamento da produção científica implicou, em primeiro lugar, na definição dos "descritores de assunto", com consulta a especialistas na área, bem como análise dos descritores disponíveis nos trabalhos anteriores, aliados ao recurso ao vocabulário controlado do Descritores em Ciências da Saúde (DeCS) e a própria estratégia de indexação da Scielo. Mediante utilização dos descritores "Política de Saúde", "Sistema Único de Saúde" e "Direito à Saúde", foram então acessados os artigos na Scielo homepage do Brasil, por abrigar em seu acervo a coleção recente de periódicos científicos brasileiros.

Foram identificados 824 artigos, dos quais foram extraídos 55 que apareceram repetidos em função do uso de três descritores correlatos. Com isso, obtivemos um total de 769 artigos publicados no período de 1988 a 2014, os quais foram submetidos a uma reclassificação a partir da leitura do título do estudo, levando-se em conta a especificidade do objeto de estudo conformea tipologia descrita no Quadro 1. Foram incluídos no primeiro grupo os estudos que tratam de análise política em saúde (politics); em um segundo conjunto, os estudos que abordam a dinâmica política em torno dos diversos componentes do sistema de saúde, quais sejam, o financiamento,a gestão/participação e controle social, os modelos de atenção, 
os recursos humanos, a informação, ciência e tecnologia em saúde; e, em um terceiro, os estudos que tratam de análises de políticas de saúde específicas (policy).

Uma vez realizada essa reclassificação, tomou-se cada um dos três conjuntos de artigos e procedeu-se a uma subdivisão temática, buscando-se identificar os subtemas abordados pelos autores. Com isso, foram construídos novos quadros que apontam o mosaico de questões selecionadas pelos diversos autores. Nessa perspectiva, os estudos que tratam de análise política ao nível macro (internacional ou nacional) foram reagrupados segundo o tema abordado. $\mathrm{O}$ mesmo foi feito com relação aos estudos sobre componentes do sistema de saúde (KLECZKOWSKI; ROEMER; VAN DER WERFF, 1984), reclassificados segundo o componente analisado. Por fim, também foram reagrupados os estudos que tratam de análise de políticas específicas, sendo que, nesse caso, adotou-se uma classificação derivada da pesquisa realizada no site do Ministério da Saúde, ${ }^{1}$ que permitiu a identificação de "políticas" elaboradas e formalizadas em documen46 tos institucionais no período de 2003-2014. (BRASIL, 2010)

Quadro 1 - Tipologia dos artigos segundo objeto de estudo. Brasil, 1988-2014

\begin{tabular}{|l|l|}
\hline \multicolumn{1}{|c|}{ Áreas } & \multicolumn{1}{c|}{ Definição } \\
\hline $\begin{array}{l}\text { 1. Análise } \\
\text { política em } \\
\text { saúde }\end{array}$ & $\begin{array}{l}\text { Política de saúde em uma perspectiva internacional; Reforma } \\
\text { Sanitária Brasileira; Processo de construção do SUS; relações } \\
\text { público-privado (SUS-SAMS). }\end{array}$ \\
\hline $\begin{array}{l}\text { 2. Componentes } \\
\text { do sistema de } \\
\text { saúde }\end{array}$ & $\begin{array}{l}\text { Financiamento da saúde (volume de recursos, formas de } \\
\text { distribuição, custos, etc,); gestão de sistemas de saúde } \\
\text { (descentralização, regionalização, modalidades alternativas de } \\
\text { gestão); participação e controle social; modelos de atenção } \\
\text { em saúde; recursos humanos em saúde; ciência, tecnologia e } \\
\text { inovação. }\end{array}$ \\
\hline
\end{tabular}

1 http://portalsaude.saude.gov.br/. 


\begin{tabular}{|l|l|}
\hline $\begin{array}{l}\text { 3. Análise } \\
\text { de políticas }\end{array}$ & $\begin{array}{l}\text { Políticas voltadas a grupos populacionais específicos (mulher, } \\
\text { criança, idoso, trabalhador etc) ou ao enfrentamento de } \\
\text { de saúde }\end{array}$ \\
específicas & $\begin{array}{l}\text { problemas específicos (aids, dengue, hipertensão arterial, } \\
\text { tuberculose etc.). }\end{array}$
\end{tabular}

Fonte: Elaborado pelas autoras.

Finalmente, foi feita uma análise do conteúdo dos títulos, resumos e palavras-chave dos 78 artigos incluídos na subárea "análise política em saúde”, com vistas a identificar a presença de expressões que indiquem a referência ao processo de RSB. Os termos utilizados nessa busca foram identificados a partir de consulta ao vocabulário controlado DeCS, no qual foi encontrado apenas o descritor "Reforma dos Serviços de Saúde" como sinônimo da RSB. Considerando que o uso dessa ferramenta pela comunidade científica é relativamente recente, optou-se por utilizar como elementos de busca além do descritor já citado, os termos: "Reforma Sanitária", "Reforma Sanitária Brasileira", "Movimento da Reforma Sanitária", "Reforma do Setor Saúde" e "Movimento Sanitário". Posto isso, os resumos dos trabalhos foram lidos utilizando a ferramenta "localizar" do Word para buscar os referidos termos no texto, extraindo-se do conjunto apenas os que continham pelo menos uma dessas expressões no título, resumo ou palavras-chave.

\section{Características e tendências da produção científica sobre política de saúde 1998-2014}

A produção científica sobre política de saúde registrada no Scielo, no período de 1988-2014, totaliza, conforme explicitado anteriormente, 769 artigos, distribuídos, segundo a data de publicação conforme a Figura 1. Verifica-se uma tendência ao crescimento do número de publicações a partir dos anos 2000, acentua-se a curva a partir do final da década, de modo que, no período 2007-2013, encontramos mais da metade das publicações (79\%). A ligeira queda 
observada em 2014, provavelmente se deve ao fato dos artigos terem sido capturados em meados do referido ano.

Figura 1 - Distribuição dos artigos por ano de publicação

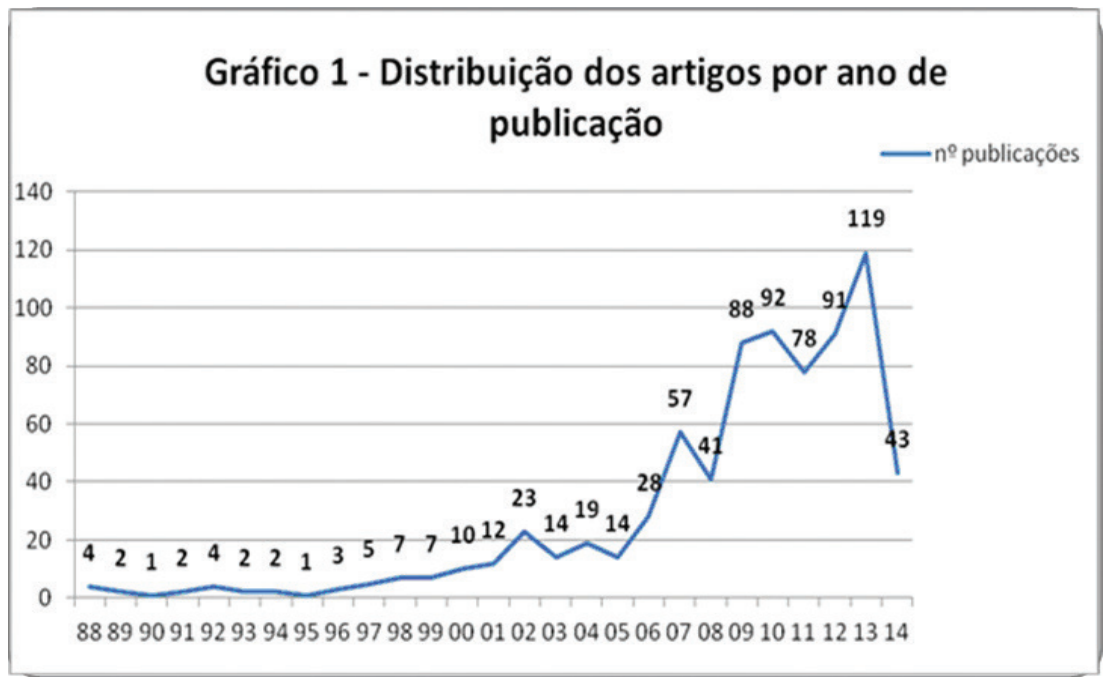

Fonte: Elaborada pelas autoras.

O aumento do número de artigos registrados na base Scielo pode estar indicando o aumento do número de periódicos que publicam trabalhos nessa área, e talvez, indiretamente, revele certo aumento do número de pesquisadores interessados nesse tema, o que, aliás, é coerente com a expansão do campo da Saúde Coletiva, especialmente dos programas de pós-graduação na área, inclusive dos mestrados profissionais (TEIXEIRA, 2006) que privilegiam a realização de estudos que abordam políticas e práticas do sistema de saúde. (ARTMANN, 2006; DOURADO et al., 2006; PIERANTONI, 2006)

A classificação do conjunto dos artigos nos grupos temáticos definidos anteriormente (Quadro 1) resultou na distribuição apresentada na Tabela 1. Do total dos estudos, 10,2\% (78) contemplam análises do processo político em saúde, enquanto 28,8\% (221) abordam aspectos referentes aos componentes do SUS. Quase metade dos trabalhos trata de análises de políticas de saúde específicas 
(49,0\%) e 12,0\% dos artigos correspondem a estudos que não se enquadram nos grupos descritos.

Tabela 1 - Distribuição dos artigos segundo objeto de estudo. Brasil, 1988-2014

\begin{tabular}{l|l|l}
\hline \multicolumn{1}{c|}{ Áreas } & \multicolumn{1}{c|}{$\mathbf{N}$} & \multicolumn{1}{c}{$\%$} \\
\hline 1. Análise política em saúde & 78 & 10,2 \\
\hline 2. Componentes do sistema de saúde & 221 & 28,8 \\
\hline 3. Análise de políticas de saúde específicas & 377 & 49,0 \\
\hline 4. Outros & 93 & 12,0 \\
\hline Total & 769 & 100,0 \\
\hline
\end{tabular}

Fonte: Elaborada pelas autoras.

\section{Distribuição dos estudos de análise política em saúde por subárea temática}

O primeiro trabalho, dentre os 78 incluídos nessa área temática, foi publicado em 1988. Durante toda a década de 1990, observou-se apenas a publicação de um trabalho por ano, sendo que, a partir do ano 2000, esse número cresceu, de modo que, no período compreendido entre 2008 e 2014, encontra-se mais da metade das publicações $(52,0 \%)$, o que evidencia, talvez, um aumento do número de pesquisadores da área que adotam uma perspectiva de análise abrangente acerca do processo político em saúde no país.

A redistribuição dos 78 artigos incluídos nesse grupo por subárea temática (Tabela 2) evidencia predominância de estudos acerca da política de saúde no Brasil (53,8\%), especialmente do processo de RSB e construção do SUS, verificando-se também estudos que abordam a política de saúde numa perspectiva internacional (20,5\%) e pequena percentagem de estudos acerca da relação entre o SUS e o Sistema de Assistência Médica Suplementar (SAMS). O restante dos trabalhos $(21,8 \%)$ trata de análise política em saúde numa perspectiva 
localizada (estudos de casos) e discussão de elementos teóricos para a análise de políticas sociais.

Esses achados evidenciam o interesse dos pesquisadores da área em acompanhar e analisar o processo de reforma do sistema de saúde ao longo dos últimos 27 anos, chamando atenção a grande quantidade de trabalhos que se focam sobre os processos que incidem sobre o sistema público, aos quais se agrega um interesse crescente na problematização das relações público-privadas, o que, de certo modo, reflete as tensões e contradições que perpassam o processo de implementação do SUS no Brasil nesse período. (PAIM, 2013a; PAIM et al., 2011; TEIXEIRA et al., 2014)

Tabela 2 - Estudos de análise política em saúde por subárea temática. Brasil, 1988-2014

\begin{tabular}{l|l|l}
\hline \multirow{2}{*}{ Subárea temática } & \multicolumn{2}{c}{ Artigos } \\
\cline { 2 - 3 } & \multicolumn{1}{c}{ No } & \multicolumn{1}{c}{$\%$} \\
\hline 1.Políticas de saúde em uma perspectiva internacional & 16 & 20,5 \\
\hline 2. Política de saúde no Brasil: RSB/SUS (Geral) & 42 & 53,8 \\
\hline 3. Relação público privado (SUS/SAMS) & 3 & 3,9 \\
\hline 4. Outros & 17 & 21,8 \\
\hline Total & $\mathbf{7 8}$ & $\mathbf{1 0 0 , 0}$ \\
\hline
\end{tabular}

Fonte: Elaborada pelas autoras.

\section{Distribuição dos estudos sobre o SUS segundo os componentes do sistema}

Sobre os componentes do sistema de saúde, foram encontrados 221 artigos (Tabela 3). A subárea temática mais frequente foi a de "gestão de sistemas e serviços de saúde" (33,4\%), seguida das subáreas "modelos de atenção à saúde" (16,7\%), "recursos humanos em saúde e ciência, tecnologia e inovação" com 15,0 \% das publicações cada uma. As subáreas "participação e controle social” (11,8\%) 
e "financiamento da saúde" apresentaram os menores números de trabalhos $(8,1 \%)$.

Tabela 3 - Estudos sobre SUS segundo componentes do sistema. Brasil, 1988-2014

\begin{tabular}{l|l|l}
\hline \multirow{2}{*}{\multicolumn{1}{c|}{ Subárea temática }} & \multicolumn{2}{c}{ Artigos } \\
\cline { 2 - 3 } \multicolumn{1}{c}{} & \multicolumn{1}{c}{ No } & \multicolumn{1}{c}{$\%$} \\
\hline a) Financiamento da saúde & 18 & 8,1 \\
\hline b) Gestão de sistemas e serviços de saúde & 74 & 33,4 \\
\hline c) Modelos de atenção à saúde & 37 & 16,7 \\
\hline d) Recursos humanos em saúde & 33 & 15,0 \\
\hline e) Ciência, tecnologia e inovação & 33 & 15,0 \\
\hline f) Participação e controle social & 26 & 11,8 \\
\hline Total & 221 & 100,0 \\
\hline
\end{tabular}

Fonte: Elaborada pelas autoras.

Essa distribuição revela o maior interesse dos pesquisadores com a problemática da gestão do sistema, abordada sob diversos ângulos, inclusive na perspectiva da chamada "gestão participativa", o que pode explicar o pequeno número de trabalhos que abordam especificamente a "participação e controle social". Chama a atenção, entretanto, o reduzido número de trabalhos sobre financiamento da saúde, um dos temas mais importantes na agenda política do setor, o que pode ter decorrido de certo viés na captura dos artigos, uma vez que não foi utilizado um descritor específico sobre esse tema.

\section{Distribuição dos estudos sobre políticas específicas do Ministério da Saúde}

Considerando que o Ministério da Saúde contempla em seu organograma, responsabilidades definidas sobre políticas específicas, os 377 artigos referentes a políticas de saúde foram distribuídos segundo essas áreas (BRASIL, 2010), a saber: "atenção à saúde”, "gestão 
do trabalho e educação da saúde", "ciência, tecnologia e insumos estratégicos", "gestão estratégica e participativa", "vigilância em saúde" e "saúde indígena" (Tabela 4).

A área de atenção à saúde é a que apresenta maior número de trabalhos (59,6\%), seguida das áreas de gestão do trabalho e educação da saúde (19,9\%), ciência, tecnologia e insumos estratégicos (10,6\%), vigilância em saúde $(7,8 \%)$, gestão estratégica e participativa $(1,6 \%)$ e, por fim, com o menor escore, a área de saúde indígena (0,5\%).

Destaca-se, na área de atenção à saúde, a predominância das publicações nas subáreas da atenção básica (19,9\%) e atenção especializada e temática (19,2\%). Na subárea "atenção básica”, a maioria dos estudos trata especificamente dessa política, provavelmente pela importância concedida à Estratégia Saúde da Família (ESF), abordada sob diversos ângulos. (TEIXEIRA; SOLLA, 2006) Em um distante segundo lugar, estão os estudos sobre a Política de Saúde Bucal, o que também parece estar vinculado à incorporação dessas ações no âmbito da atenção básica, principalmente nos últimos 12 anos. (SOUZA; RONCALLI, 2007)

Há predominância na subárea "ações programáticas e estratégicas" de estudos sobre a Política Nacional de Atenção Integral à Saúde da Mulher (6,9\%) e em segundo lugar sobre a Política Nacional de Atenção Integral à Saúde da Criança e Aleitamento Materno (4,8\%), o que pode refletir a prioridade tradicionalmente atribuída a esses dois grupos na atenção primária à saúde, embora o modelo empregado preconize uma abordagem familiar. (SANTOS NETO, 2008)

Observa-se na subárea "regulação, avaliação e controle de sistemas", o menor número de estudos $(0,8 \%)$, os quais abordavam aspectos dos sistemas de informação e controle de sistemas e serviços em detrimento da regulação e avaliação, planejamento e programação das ações, não abordados nos estudos.

Verifica-se, na subárea "atenção hospitalar e urgência” (3,2\%), discreta concentração de estudos acerca da Política Nacional de Atenção Hospitalar (1,3\%), tema que parece estar sendo abordado mais em função da relevância da atenção hospitalar ser o compo- 
nente do modelo assistencial responsável pela maior absorção de recursos (CARVALHO, 2007) do que do ponto de vista da política governamental. Já a existência de estudos sobre a Política Nacional de Transplantes de Órgãos e Tecidos (1,1\%), ainda que em número reduzido, sinaliza a importância do Programa de Doação, Captação e Transplante de Órgãos e Tecidos no Brasil, o qual realiza todos os tipos de transplantes com financiamento do SUS, incluindo oferta gratuita da medicação necessária após a realização das cirurgias, fazendo com que o Brasil possua atualmente o maior programa público de transplantes do mundo. (MARINHO, 2006)

Componente da área de "atenção à saúde", a Política Nacional de Humanização foi abordada em 4\% dos estudos, percentual esse superior à subárea anteriormente citada, tal achado demonstrando a relevância que vem sendo atribuída à mudança nos modos de gerir e cuidar em saúde, que motivou a criação da Política Nacional de $\mathrm{Hu}$ manização em 2003. (PASCHE; PASSOS; HENNINGTON, 2011)

Segunda área com maior número de publicações (19,9\%), a "gestão do trabalho e educação da saúde" concentra-se em estudos da Gestão da Educação na Saúde (17,2\%) em comparação à Política Nacional de Educação Permanente em Saúde que foi estudada em apenas 2,7\% dos trabalhos. Tal achado sinaliza maior dedicação dos estudos relacionados à formação profissional em saúde em detrimento da educação permanente dos profissionais e trabalhadores do setor, tema só mais recentemente priorizado na agenda política do sistema.

$\mathrm{Na}$ área de ciência, tecnologia e insumos estratégicos, foi encontrada percentagem quatro vezes superior de estudos sobre a Política Nacional de Assistência Farmacêutica (8,5\%), o que indica a relevância que a assistência farmacêutica tem assumido no SUS nos últimos anos na tentativa de melhorar o acesso dos brasileiros aos fármacos de que necessitam, podendo-se citar iniciativas como o Programa de Farmácia Popular criado em 2004 e sua expansão para a Rede Privada em 2006. (VIEIRA, 2010)

Contudo, o acesso ainda é limitado, motivando inclusive onda crescente de ações judiciais para fornecimento de medicamentos e 
produtos, que integram o contemporâneo fenômeno da "judicialização da saúde”. (PEPE et al., 2010) O pequeno número de estudos sobre Política Nacional de Ciência, Tecnologia e Inovação em Saúde $(2,1 \%)$, por sua vez, pode estar refletindo o mesmo processo indicado para a relação Gestão da Educação na Saúde/Educação permanente, ou seja, essa temática só ganhou relevância da agenda política nos últimos 12 anos, o que provavelmente vem estimulando a realização de estudos acerca da incorporação de tecnologias e inovações no SUS.

Tabela 4- Distribuição dos artigos segundo políticas de saúde específicas. Brasil, 1988-2014

\begin{tabular}{|c|c|c|}
\hline Artigos por áreas, subáreas temáticas e políticas & $\mathbf{N}$. & $\%$ \\
\hline Atenção à saúde & 225 & 59,6 \\
\hline 1.1 Atenção básica & 75 & 19,9 \\
\hline 1.1.1 Política Nacional de Atenção Básica & 50 & 13,3 \\
\hline 1.1.2 Política Nacional de Saúde Bucal & 13 & 3,4 \\
\hline 1.1.3 Política Nacional de Alimentação e Nutrição & 2 & 0,5 \\
\hline $\begin{array}{l}\text { 1.1.4 Política Nacional de Práticas Integrativas e } \\
\text { Complementares }\end{array}$ & 10 & 2,7 \\
\hline 1.2Atenção especializada e temática & 73 & 19,2 \\
\hline 1.2.1 Política Nacional de Saúde da Pessoa Idosa & 05 & 1,3 \\
\hline 1.2.2 Política Nacional de Média e Alta Complexidade & 19 & 5,0 \\
\hline $\begin{array}{l}\text { 1.2.3 Política/Programa de Atenção às Pessoas com } \\
\text { Doenças Crônicas }\end{array}$ & 13 & 3,4 \\
\hline 1.2.4 Política Nacional de Saúde Mental & 27 & 7,2 \\
\hline $\begin{array}{l}\text { 1.2.5 Política de Atenção Integral aos Usuários de Álcool e } \\
\text { Outras Drogas }\end{array}$ & 4 & 1,0 \\
\hline $\begin{array}{l}\text { 1.2.6 Política Nacional de Atenção Integral à Saúde do } \\
\text { Homem }\end{array}$ & 5 & 1,3 \\
\hline 1.3 Ações programáticas e estratégicas & 47 & 12,5 \\
\hline $\begin{array}{l}\text { 1.3.1 Política Nacional de Atenção Integral à Saúde das } \\
\text { Pessoas Privadas de Liberdade no Sistema Prisional }\end{array}$ & 0 & 0,0 \\
\hline
\end{tabular}




\begin{tabular}{|c|c|c|}
\hline $\begin{array}{l}\text { 1.3.2 Política Nacional de Atenção Integral à Saúde da } \\
\text { Mulher }\end{array}$ & 26 & 6,9 \\
\hline $\begin{array}{l}\text { 1.3.3 Política Nacional de Atenção Integral à Saúde da } \\
\text { Criança e Aleitamento Materno }\end{array}$ & 18 & 4,8 \\
\hline $\begin{array}{l}\text { 1.3.4 Política Nacional de Atenção Integral à Saúde do } \\
\text { Adolescente }\end{array}$ & 1 & 0,3 \\
\hline 1.3.5 Política Nacional de Saúde da Pessoa com Deficiência & 2 & 0,5 \\
\hline 1.4 Regulação, avaliação e controle de sistemas & 3 & $\mathbf{0 , 8}$ \\
\hline 1.4.1 Sistemas de informação & 2 & 0,5 \\
\hline 1.4.2 Controle de serviços e sistemas & 1 & 0,3 \\
\hline 1.4.3 Regulação e svaliação & 0 & 0,0 \\
\hline 1.4.4 Planejamento e programação das ações em saúde & 0 & 0,0 \\
\hline 1.5 Atenção hospitalar e urgência & 12 & 3,2 \\
\hline $\begin{array}{l}\text { 1.5.1 Política Nacional de Sangue, Componentes e } \\
\text { Hemoderivados }\end{array}$ & 1 & 0,3 \\
\hline 1.5.2 Rede de Atenção às Urgências e Emergências & 2 & 0,5 \\
\hline $\begin{array}{l}\text { 1.5.3 Política Nacional de Transplantes de Órgãos e } \\
\text { Tecidos }\end{array}$ & 4 & 1,1 \\
\hline 1.5.4 Política Nacional de Atenção Hospitalar & 5 & 1,3 \\
\hline 1.6 Política Nacional de Humanização & 15 & 4,0 \\
\hline Gestão do trabalho e educação na saúde & 75 & 19,9 \\
\hline 2.1 Gestão da educação na saúde & 65 & 17,2 \\
\hline 2.2 Política Nacional de Educação Permanente em Saúde & 10 & 2,7 \\
\hline Ciência, tecnologia e insumos estratégicos & 40 & 10,6 \\
\hline 3.1 Política Nacional de Assistência Farmacêutica & 32 & 8,5 \\
\hline $\begin{array}{l}\text { 3.2 Política Nacional de Ciência, Tecnologia e Inovação em } \\
\text { Saúde }\end{array}$ & 8 & 2,1 \\
\hline Gestão estratégica e participativa & 06 & 1,6 \\
\hline $\begin{array}{l}\text { 4.1 Política Nacional de Gestão Estratégica e Participativa no } \\
\text { SUS }\end{array}$ & 2 & 0,5 \\
\hline 4.2 Política Nacional de Educação Popular em Saúde & 1 & 0,3 \\
\hline
\end{tabular}




\begin{tabular}{c|l|l}
\hline 4.3 Política Nacional de Saúde Integral da População Negra & 3 & 0,8 \\
\hline $\begin{array}{l}\text { 4.4 Política Nacional de Saúde Integral de Outras Populações } \\
\text { (Lésbicas, Gays, Bissexuais, Travestis e Transexuais; Campo e } \\
\text { floresta; em Situação de rua e Cigana }\end{array}$ & 0 & 0,0 \\
\hline Vigilância em saúde & $\mathbf{2 9}$ & $\mathbf{7 . 8}$ \\
\hline 5.1 Vigilância epidemiológica & $\mathbf{0 7}$ & $\mathbf{1 , 9}$ \\
\hline 5.1.1 Programa Nacional de Controle da Tuberculose & 3 & 0,8 \\
\hline $\begin{array}{c}\text { 5.1.2 Programa Nacional de Imunizações, Controle } \\
\text { da Hanseníase, da Dengue, da Malária }\end{array}$ & 0 & 0,0 \\
\hline 5.1.3 Controle de chagas e esquistossomose & 4 & 1,1 \\
\hline 5.2 Análise de situação de saúde & $\mathbf{7}$ & $\mathbf{1 , 9}$ \\
\hline 5.2.1 Política Nacional de Promoção da Saúde & $\mathbf{7}$ & $\mathbf{1 , 9}$ \\
\hline $\begin{array}{l}\text { 5.3 Vigilância, prevenção e controle das doenças } \\
\text { sexualmente transmissíveis, síndrome da } \\
\text { imunodeficiência adquirida e hepatites virais }\end{array}$ & $\mathbf{0 8}$ & $\mathbf{2 , 1}$ \\
\hline $\begin{array}{l}\text { 5.3.1 Política Nacional de Prevenção de DST/HIV/AIDS e } \\
\text { Hepatites Virais }\end{array}$ & 8 & 2,1 \\
\hline 5.4 Vigilância em saúde ambiental e do trabalhador & $\mathbf{0 7}$ & $\mathbf{1 , 9}$ \\
\hline 5.4.1 Política Nacional de Saúde Ambiental & 1 & 0,3 \\
\hline $\begin{array}{l}\text { 5.4.2 Política Nacional de Saúde do Trabalhador } \\
\text { Saúde indígena }\end{array}$ & 6 & 1,6 \\
\hline 6.1 Política Nacional de Atenção à Saúde dos Povos Indígenas & 2 & 0,5 \\
\hline Total & $\mathbf{3 7 7}$ & $\mathbf{1 0 0 , 0}$ \\
\hline
\end{tabular}

Fonte: Elaborada pelas autoras.

$\mathrm{Na}$ área de gestão estratégica e participativa, predominam estudos acerca da Política Nacional de Saúde Integral da População Negra em detrimento de outros grupos $(0,8 \%)$, o que pode estar relacionado à representatividade e consolidação do Movimento Social Negro. (LIMA, 2010) Chama a atenção o fato de ter aparecido apenas um estudo sobre a Política Nacional de Educação Popular em Saúde e não aparecerem publicações sobre várias outras políticas que 
têm sido formuladas mais recentemente, como é o caso da Política Nacional de Saúde Integral de outras populações (Lésbicas, Gays, Bissexuais, Travestis e Transexuais; Campo e floresta; em Situação de rua e Cigana).

$\mathrm{Na}$ área de vigilância em saúde, estudos sobre a Política Nacional de Prevenção de DST/HIV/AIDS e Hepatites Virais foram os mais frequentes $(2,1 \%)$, sugerindo a relevância atribuída à temática no país que conta com movimento social organizado em torno do tema e um programa que, embora tenha dividido opiniões quanto à sua efetividade, destaca-se no mundo por oferecer tratamento antirretroviral gratuito. (VILLARINHO et al., 2013) Em segundo lugar, aparecem os estudos acerca da Política Nacional de Promoção da saúde $(1,9 \%)$, tema que tem sido abordado por vários pesquisadores, sendo que a divulgação dos resultados desses estudos não tem se dado através de artigos científicos e sim através de livros e capítulos de livro. (PELLEGRINI FILHO; BUSS; ESPERIDIÃO, 2014; TEIXEIRA, 2006) Foi encontrado também um único estudo sobre Política Nacional de Saúde Ambiental e não houve publicações sobre os Programas Nacionais de Imunizações e Controle da Hanseníase, da Dengue e da Malária.

Por fim, a área de saúde indígena foi contemplada com apenas 0,5\% das publicações. Embora corresponda a parcela numericamente pouco expressiva da população, os índios têm grande importância histórico-cultural para o país, o que poderia motivar parcela mais expressiva de estudos dedicados às suas diversas demandas, muito específicas, relativas à sua sobrevivência física e cultural diante da acelerada e complexa transformação social a que foram submetidos. (CHAVES; CARDOSO; ALMEIDA, 2006)

\section{Análise de conteúdo dos artigos de "análise política em saúde"}

Além da análise da distribuição dos artigos selecionados segundo temas e objetos de estudo, realizamos a análise dos 78 artigos in- 
cluídos na subárea "análise política em saúde” (Tabela 1) com vistas a identificar a presença ou não de referência à RSB, e caso positivo, em que período situam esse processo. Do conjunto de trabalhos analisados, apenas 16 fazem menção à RSB ou expressões correlatas, aparecendo como palavra-chave o termo "Reforma Sanitária" (9), e os termos "Movimento Sanitário" (1), "Reforma do Setor Saúde" (1), "Reforma dos Serviços de Saúde" (1), sendo que, nos demais (4), os termos aparecem apenas no texto do resumo ou no título do artigo.

Quanto à evolução temporal, apenas um trabalho foi publicado na década de 1990 (QUEIROZ; VIANNA, 1992), sendo os demais publicados a partir dos anos 2000. A análise de conteúdo desses artigos revela que a grande maioria (15) faz menção direta à RSB seja em seus títulos, objetivos ou resumos. Muitos deles contemplam algum resgate histórico do movimento da RSB, suas origens históricas, bases conceituais e características do processo político. (CAMPOS, 2007b; COELHO, 2010; CORDEIRO, 2004; FLEURY, 2009; MERHY, 2012; PAIM, 2006, 2008b; PAIVA; TEIXEIRA, 2014; QUEIROZ; VIANNA, 1992; SANTOS, 2013; SANTOS; SILVA, 2013) Destes, apenas seis fazem menção à permanência do processo de RSB na conjuntura mais recente. (CAMPOS, 2007b; COHN, 2009; FLEURY, 2009; PAIM, 2006, 2008b; SANTOS, 2013) Cabe assinalar que o artigo incluído nesse grupo que não utiliza a expressão RSB ou "reforma sanitária”, usa o descritor "reforma do setor saúde”, assumindo uma perspectiva de análise de políticas comparadas ao discutir elementos centrais desse processo em países da América Latina. (ALMEIDA, 2002)

Chama a atenção o fato de que alguns dos autores que abordam o processo de RSB são reconhecidos como militantes históricos do movimento sanitário, conforme, aliás, é apontado por um dos trabalhos selecionados (PAIVA; TEIXEIRA, 2014) e apresentam uma reflexão fundamentada na avaliação dos avanços e dificuldades enfrentadas no processo. Um primeiro grupo se atém ao debate acerca da natureza do processo de RSB e identifica os desafios políticos e institucionais para sua continuidade e aperfeiçoamento. Outros, entretanto, apesar de se 
referirem à $\mathrm{RSB}$, concentram sua análise no processo de construção do SUS, aspecto que, aliás, não passa despercebido dos primeiros, porém não é o foco central da sua análise.

Dos autores que situamos no primeiro grupo, destacam-se Jairnilson Paim, Gastão Wagner de Sousa Campos, Sonia Fleury e Nelson Rodrigues dos Santos. Paim (2008b, p. 640), aponta que apesar de suas notáveis conquistas, “[...] a RSB restringiu-se a uma reforma parcial de natureza setorial e institucional traduzida pelos 20 anos de implementação tortuosa do SUS”. As limitações da RSB também são reconhecidas por Fleury (2009), ao afirmar que, a política pública institucionalizada a partir da RSB, o [...] instituído, “[...] se impôs ao instituinte, reduzindo o caráter libertário e transformador da reforma [...]". Para a autora, "o paradoxo da reforma sanitária brasileira é que seu êxito [...] reduziu a capacidade de ruptura, inovação e construção de uma nova correlação de forças desde a sociedade civil organizada [...]”. (FLEURY, 2009, p. 751) Outro autor que analisa as dificuldades desse processo é Campos (2007b, p. 1873), para quem a concretização da RSB enfrenta barreiras, como por exemplo, a “[...] cultura patrimonialista predominante na administração pública [...]"e a falta de eficiência das Conferências e dos Conselhos de Saúde, que apesar de relevantes, não tem sido suficientes para “[...] indicar rumos e criar os consensos [...]".

Como se pode constatar, os autores acima citados, apesar das diferenças na abordagem do fenômeno "reforma sanitária brasileira", têm em comum a compreensão de que esse processo não se encontra necessariamente encerrado, considerando que, enquanto movimento social, a RS “[...] está desafiada a persistir na sua ação inovadora e criativa, na luta pelos direitos sociais". (SANTOS, 2013, p. 278)

Para isso, Paim (2008b, p. 627) considera que a análise do significado e da viabilidade do projeto da RSB exige a realização de investigações que permitam a identificação de "[...] atores sociais relevantes" capazes de manter e dar continuidade ao processo. Nessa perspectiva, Santos (2013, p. 278-279), defende a 
[...] retomada da comunicação direta com as entidades da sociedade e movimentos sociais; [...] fortalecimento dos conselhos de saúde; e [...] superação dos embates entre as causalidades governamentais e partidárias de um lado, e de outro, a conscientização e mobilização supra e apartidária por reforma do Estado, democrática e política, voltada para as diretrizes constitucionais de políticas públicas para os direitos sociais [...] .

Sonia Fleury (2009, p. 751), por sua vez, aponta alguns desafios institucionais presentes no momento atual da reforma sanitária, quais sejam:

[...] a incapacidade de implantar um modelo integral de atenção à saúde $[. .$.$] a incapacidade de implantar melho-$ rias na gestão do sistema e na gestão das unidades, $[. .$.$] a$ falta de uma renovação ética dos profissionais de saúde e [...] a dependência de insumos e medicamentos cujos preços e condições de produção por grandes empresas multinacionais fogem ao controle dos Estados nacionais [...].

Em outra perspectiva, situam-se os autores que analisam a RSB sob a ótica do processo de construção do SUS, destacando-se Amélia Cohn, para quem "o processo de implantação do SUS [...], induziu - com todos os seus méritos [...] - os militantes da área a abdicarem da Reforma Sanitária enquanto um projeto emancipatório”. (COHN, 2009, p.1616) Para a autora, talvez o que esteja ocorrendo na atualidade, seja uma "reforma da reforma", num processo em que a "[...] dimensão da política vem sendo subsumida pela dimensão técnico-cientifica, levando a uma pasteurização da própria dimensão política”. (COHN, 2009, p. 1618) A hipótese da autora é que a explicação para tal fato deriva da "[...] incapacidade da comunidade do campo sanitário de formular um novo projeto para saúde articulado com um projeto para a sociedade”. (COHN, 2009, p. 1618)

Cardoso e Campos (2013, p. 221-222), por sua vez, destacam que o 
[...] alcance incompleto e descontínuo das conquistas do movimento de reforma sanitária se relaciona à inviabilidade da estratégia de conquistas progressivas e cumulativas de direitos universais no interior do Estado brasileiro [...]. Tal Estado, marcado pela influência do grande capital sobre todas as esferas da sociedade e do poder estatal, bem como pelo ataque permanente aos direitos da classe trabalhadora [...], escancara os estreitos limites do capitalismo dependente. Assim, [...] as reivindicações em torno da 'democratização progressiva', bandeiras do movimento de luta pela reforma sanitária, se chocaram com a ordem social e política brasileira.

Coerentemente com o foco de sua análise, Cohn (2009, p. 1618) defende que a superação desses desafios implica em se buscar "[...] identificar quem são e onde se constroem novas redes de sociabilidade e novas identidades sociais" capazes de levar à frente esse projeto, perspectiva que, de certo modo, conflui com as propostas elaboradas por Paim (2013b, p. 1953) com relação à identificação dos "sujeitos da antítese" capazes de dar continuidade ao processo de RSB e também com a proposta de Fleury (2009, p. 751), acerca da necessidade de “[...] construção permanente do sujeito, aquele que poderá transformar novamente o instituído em instituinte, para de novo institucionalizar-se".

Por outro lado, Campos (2007a) elenca em seu estudo sete estratégias que se concentram, entretanto, na busca de consolidar o SUS. Para esse autor, o caminho para avançar a reforma sanitária depende, de se

[...] privilegiar, no consenso a ser instaurado, elementos da tradição dos sistemas públicos, procurando, com isto, resolver uma série de ambigüidades discursivas e pragmáticas, que tem permitido ao ideário liberal-privatista sobreviver para além do conveniente e do necessário. (CAMPOS, 2007b, p. 1873) 
Como vemos, apesar da confluência de concepções acerca da $\mathrm{RSB}$, os trabalhos analisados revelam a diferença de propostas e estratégias para se garantir a viabilidade política bem como a continuidade do processo de RSB no âmbito institucional.

\section{Considerações finais}

O mapeamento da produção científica sobre política de saúde no período 1988-2014 é uma primeira aproximação que será desdobrada em estudos posteriores que identifiquem as abordagens teóricas utilizadas pelos diversos autores. $\mathrm{O}$ estudo, entretanto, revela algumas características da prática de pesquisa que vem sendo desenvolvida na área de política, planejamento e gestão em saúde, e estimula o levantamento de propostas para a continuidade e aprofundamento da análise dos trabalhos publicados nessa área.

Em primeiro lugar, observa-se uma tendência ao aumento da quantidade de artigos publicados ao longo do período, o que certamente reflete o aumento exponencial de grupos de pesquisa nessa área, os quais somavam, em abril de 2015, cerca de 81 grupos registrados na plataforma de grupos de pesquisa do Conselho Nacional de Desenvolvimento Científico e Tecnológico (CNPq). Como apontamos anteriormente, tal fato pode estar ligado, inclusive, ao aumento do número de programas de cursos de pós-graduação na área de Saúde Coletiva, inclusive de mestrados profissionalizantes.

Em segundo lugar, chama atenção a mudança que vem se verificando na distribuição desses trabalhos por área temática, o que evidencia certa redefinição dos objetos de investigação e, provavelmente, uma diversificação de abordagens teórico-metodológicas utilizadas na análise dos diversos temas. De fato, no levantamento feito 10 anos atrás (TEIXEIRA; PAIM, 2005), observou-se que, na maioria dos estudos sobre política de saúde realizados até o início da década de 1990, predominava uma perspectiva macro, utilizando-se categorias extraídas da abordagem marxista, tendo como referencial as relações Estado-Sociedade, considerados determinantes dos pro- 
cessos políticos em saúde. Paulatinamente, foram surgindo investigações sobre políticas, instituições e práticas de saúde, valorizandose, também, estudos de avaliação de políticas e programas de saúde.

Essa tendência parece ter se intensificado nos últimos anos, uma vez que o mapeamento do conjunto da produção revela a concentração dos estudos na análise de políticas específicas, o que pode estar refletindo a própria fragmentação do processo de formulação e implementação de políticas de saúde no Brasil, com ênfase na elaboração de propostas de intervenção sobre problemas apresentados por grupos populacionais específicos, que, ao se organizarem politicamente e ocuparem espaços de decisão no âmbito governamental, passam a pressionar pela formulação e implementação de programas e ações especificamente dirigidas ao atendimento de suas demandas.

Também chama a atenção o crescimento de estudos que abordam o processo de construção do SUS, tendencialmente voltados à análise de componentes específicos do sistema, principalmente os aspectos relacionados à gestão do sistema, seguido de estudos sobre modelos de atenção, financiamento, ciência e tecnologia, recursos humanos e participação social. Nessa perspectiva, é de se esperar a incorporação de referenciais teóricos provindos de disciplinas específicas, como é o caso da administração pública, economia, educação etc., colocando-se, portanto, a necessidade de se avançar com a leitura e análise dos textos completos.

Chama a atenção, por fim, o número relativamente reduzido de estudos que abordem as questões do poder em saúde na perspectiva macropolítica, da relação entre Estado e as classes sociais no Brasil contemporâneo, especialmente tendo em vista a problematização da sustentabilidade política do processo da RSB e os determinantes das tendências atuais de valorização do mercado de serviços de saúde, expressas tanto na expansão dos planos de saúde e serviços privados quanto na privatização do sistema público. (COSTA; BAHIA; SCHEFFER, 2013; SCHEFFER, 2015; SESTELO; SOUZA, BAHIA; 2013) Como vimos, são poucos os estudos que problematizam a RSB, quer analisando as dificuldades enfrentadas no 
processo, quer apontando estratégias para garantir sua viabilidade e continuidade na conjuntura mais recente. É possível que isso reflita, indiretamente, a diversidade de concepções acerca da vitalidade do processo da RSB (HOCHMAN, 2013; PAIM, 2013b), com grande parte dos estudiosos se ocupando muito mais dos processos institucionais de construção do SUS do que da luta política mais geral para viabilizar a RSB enquanto uma reforma social, uma mudança no "modo de vida". (PAIM, 2008a)

Isso reforça a necessidade de se avançar na leitura dos textos completos desses trabalhos, de modo a identificar a filiação dos autores a determinadas correntes de pensamento, tendo como referência o debate político mais geral sobre os rumos da sociedade brasileira no contexto atual e as perspectivas que se desenham em termos de política de saúde. Do mesmo modo, isso também pode vir a ser feito com os trabalhos que abordam os diversos componentes do SUS e as políticas específicas, contribuindo para subsidiar as investigações em curso nos diversos eixos temáticos do Observatório de Análise

64 Política em Saúde (OAPS), cuja finalidade principal é subsidiar o debate sobre os modos de produzir conhecimento cientificamente fundamentado e politicamente comprometido com o aperfeiçoamento do processo de construção do SUS e da melhoria da efetividade das ações voltadas ao enfrentamento dos problemas de saúde dos distintos grupos populacionais. Esse é exatamente o desafio que estamos tratando de enfrentar como parte da pesquisa que vem sendo desenvolvida no eixo 1 do OAPS, que trata especificamente do monitoramento do processo político em saúde, com base no acompanhamento de notícias veiculadas nos sites de diversos órgãos governamentais e não governamentais, envolvidos direta ou indiretamente com o avanço do processo de Reforma Sanitária no país.

\section{Referências}

AGENDA estratégica para a saúde no Brasil. 2011. Disponível em: <http://www6.ensp.fiocruz.br/radis/sites/default/files/109/pdf/ agenda-saude-para-todos.pdf $>$. Acesso: 21 jul. 14 . 
(UMA) AGENDA estratégica para a saúde no Brasil.[S.l.], 2011.

Disponível em: <http://www.cebes.org.br/media/File/ Agenda\%20 Estrategica \%20para\%20a\%20Saudepdf|>. Acesso em: 19 jul. 2014.

ALMEIDA, C. Eqüidade e reforma setorial na América Latina:um debate necessário. Caderno Saúde Pública, Rio de Janeiro, n. 18, p. 23-36, 2002. Suplemento.

ARTMANN, E. Desafios na formação de Recursos Humanos para o SUS: a experiência do Mestrado profisisonalizante da ENSP com a SAS-MS. In: LEAL, M.C.; FREITAS, C. M. (Org.). Cenários possíveis: experiências e desafios do mestrado profissional na saúde coletiva. Rio de Janeiro: Ed. Fiocruz, 2006. p. 151-189.

BAHIA, L. A saúde em banho-maria. In: MAGALHÃES, J. P. de A. Os anos Lula: contribuições para um balanço crítico 2003-2010. Rio de Janeiro: Garamond, 2010. p. 351-368.

BORGES, F. T. et al. Anatomia da privatização neoliberal do Sistema Único de Saúde: o papel das Organizações Sociais de Saúde. São Paulo: Cultura Acadêmica, 2012.

BRASIL . Conselho Nacional de Saúde. $8^{a}$ Conferência Nacional de Saúde: relatório final. Brasília, DF, 1986a.

BRASIL. Constituição (1988). Constituição da República Federativa do Brasil. Brasília, DF: Senado, 1988.

BRASIL. Ministério da Saúde. Portaria Interministerial MEC/MS/ MPAS N. 9 02/86. Diário Oficial da União. Brasilia, DF, 22 ago. 1986b.

BRASIL. Ministério da Saúde. Secretaria Executiva. Coordenação Geral de Inovação Gerencial. Regimentos Internos e organogramas básicos do Ministério da Saúde Brasília, DF, 2010. (Série E. Legislação de saúde).

CAMPOS, G. W. de S. Reforma política e sanitária:a sustentabilidade do SUS em questão? Ciência e Saúde Coletiva, Rio de Janeiro, v. 12, n. 2, p. 301-306, abr. 2007a.

CAMPOS, G. W. de S. O SUS entre a tradição dos Sistemas Nacionais e o modo liberal-privado para organizar o cuidado à saúde. Ciência Saúde e Coletiva, Rio de Janeiro, v. 12, p. 1865-1874. nov. 2007b. Suplemento. 
CARDOSO, F. M.; CAMPOS, G. W. de S. Reformas Neoliberais, Reforma Sanitária Brasileira e Fundações Estatais de Direito Privado: análise de documento da Fundação Estatal de Saúde da Família (FESF) Bahia. Saúde em debate, Rio de Janeiro, v. 37, n. 97, p. 219-232, Jun. 2013.

CARVALHO, D. M. T. de. Financiamento da assistência médicohospitalar no Brasil. Ciência Saúde e Coletiva, Rio de Janeiro, v. 12, n. 4, ago. 2007.

CHAVES, M. de B. G.; CARDOSO, A. M.; ALMEIDA, C. Implementação da política de saúde indígena no Pólo-base Angra dos Reis, Rio de Janeiro, Brasil: entraves e perspectivas.Caderno Saúde Pública, Rio de Janeiro, v. 22, n. 2, p. 295-305, fev. 2006.

COELHO, I. B. Democracia sem equidade: um balanço da reforma sanitária e dos dezenove anos de implantação do Sistema Único de Saúde no Brasil. CiênciaSaúde Coletiva, Rio de Janeiro, v. 15, n.1, p. 171-183, jan. 2010

COHN, A. A reforma sanitária brasileira após 20 anos do SUS: reflexões. Caderno Saúde Pública, Rio de Janeiro, v. 25, n. 7, p. 1614-1619, jul. 2009. CORDEIRO, H. O Instituto de Medicina Social e a luta pela reforma sanitária: contribuição à história do SUS. Physis: Revista de Saúde Coletiva, Rio de Janeiro v. 14, n. 2, p. 343-362, jul./dez. 2004.

CÔRTES, S. V. Conselhos e conferências de saúde: papel institucional e mudança nas relações entre Estado e sociedade. In: LOBATO, L. de V. C; FLEURY; S. Participação, democracia e saúde. Rio de Janeiro: CEBES, 2010, p. 102-128. (Coleção Pensar em Saúde).

CÔRTES, S. V. Sistema Único de Saúde: espaços decisórios e a arena política de saúde. Cadernos de Saúde Pública, Rio de Janeiro, v. 25, n. 7, p. 1626-1633, jun. 2009.

COSTA, A. M.; BAHIA, L.; SCHEFFER, M. Onde foi parar o sonho do SUS? Le Monde Diplomatique, São Paulo, n. 69, p. 30-31, 2013.

DOURADO, I. et al. A experiência do mestrado profissional do Instituto de Saúde Coletiva da UFBA 2001-2004. In: LEAL, M. C.; FREITAS, C. M. (Org.). Cenários possíveis: experiências e desafios do mestrado 
profissional na saúde coletiva. Rio de Janeiro: Ed.Fiocruz, 2006.

p. 101-121.

ESCOREL, S. Reviravolta na saúde: origem e articulação do movimento sanitário. Rio de Janeiro: Ed. Fiocruz, 1999.

FLEURY, S. Estado sem cidadãos: seguridade social na América Latina. Rio de Janeiro: Ed. Fiocruz, 1994.

FLEURY, S. Reforma sanitária brasileira: dilemas entre o instituinte e o instituído.Ciência e Saúde Coletiva, Rio de Janeiro, v. 14, n. 3, p. 743-752, Jun. 2009.

GERSCHMAN, S. A democracia inconclusa: um estudo da reforma sanitária brasileira. Rio de Janeiro: Ed. Fiocruz, 1985.

HOCHMAN, G. Saudades do futuro ou um sistema de saúde em tempos democráticos. Caderno Saúde Pública, Rio de Janeiro, v. 29, n. 10, p. 1927-1953, out. 2013.

KINGDON, J. W. Agendas, alternatives, and public policies. $3^{\text {rd }}$. Washington: LONGMAN, 2011.

KLECZKOWSKI, B.; ROEMER, M.; VAN DER WERFF, A. Sistemas nacionales de salud y su reorientación hacia la salud para todos. Cuadernos de Salud Pública, La Habana, n. 77, 1984.

LABRA, M. H.; FIGUEIREDO, J. S. A de. Associativismo, participação e cultura cívica: o potencial dos conselhos de saúde. Ciência e Saúde Coletiva, Rio de Janeiro, v. 7, n. 3, p. 537-547, 2002.

LEVCOVITZ, E. et al. Produção de conhecimento em política, planejamento e gestão em saúde e políticas de saúde no Brasil (1974-2000). Brasília, DF: OPAS, 2003. (Série Técnica Projeto de Desenvolvimento de Sistemas de Serviços de Saúde, 2).

LIMA, M. Desigualdades raciais e políticas públicas: ações afirmativas no governo Lula. Novos Estudos - CEBRAP, São Paulo, n. 87, p. 77-95, July 2010.

LIMA, N. T; SANTANA, J. P. (Org.). Saúde coletiva como compromisso: a trajetória da ABRASCO. Rio de Janeiro: Ed. Fiocruz, 2006. 
LOBATO, L. de V. C; FLEURY; S. Participação, Democracia e Saúde.Rio de Janeiro: CEBES, 2009.(Coleção Pensar em Saúde).

MACHADO, C.V.; BAPTISTA, T.W.F.; LIMA, L.D. (Org.). Políticas de Saúde no Brasil: continuidades e mudanças. Rio de Janeiro: Ed. Fiocruz, 2012.

MAGNO, L. D. Dos clamores das ruas aos rumores no congresso: uma análise da conjuntura recente da saúde no Brasil. 2014. 105 f. Dissertação (Mestradoem Saúde Coletiva) -Intituto de SaudeColetiva, Universidade Federal da Bahia, Salvador, 2014. Disponível em: <http://repositorio. ufba.br/ri/handle/ri/18272>. Acesso em: 20 mar. 16.

MARINHO, A. Um estudo sobre as filas para transplantes no Sistema Único de Saúde brasileiro.Cadernode Saúde Pública,Rio de Janeiro, v. 22, n. 10, p. 2229-2239, Oct. 2006.

MERHY, E. Saúde e direitos: tensões de um SUS em disputa, molecularidades.Saúde e Sociedade, São Paulo, v. 21, n. 2, p. 267-279, Jun. 2012.

NORONHA, J.C.; LIMA, L.D.; MACHADO, C.V. O Sistema Único de Saúde. In: GIOVANELLA, L. et al. (Org.). Políticas e sistemas de saúde no Brasil. 2. ed. Rio de Janeiro: Ed. Fiocruz, 2012. p. 365-393.

NORONHA, J.C.; SANTOS, I.; PEREIRA, T. Relações entre o SUS e a saúde suplementar: problemas e alternativas para o futuro do sistema universal. In: SANTOS, N.R.; AMARANTE, P.D.C. (Org.). Gestão pública e relação público-privado na saúde. Rio de Janeiro: Cebes, 2011. p. 152-179.

OCKÉ-REIS, C.O. SUS: o desafio de ser único. Rio de Janeiro:Ed. Fiocruz, 2012.

PAIM, J. S. A Constituição cidadã e os 25 anos do Sistema Único de Saúde. Caderno Saúde Pública, Rio de Janeiro, v. 29, n. 10, p. 1927-1953, 2013a.

PAIM, J. S. Eqüidade e reforma em sistemas de serviços de saúde: o caso do SUS. Saúde e Sociedade, São Paulo, v. 15, n. 2, p. 34-46, ago. 2006. 
PAIM, J. S. O futuro do SUS. Caderno de Saúde Pública, Rio de Janeiro, v. 28, p. 612-613, 2012.

PAIM, J. S. Políticas de saúde no Brasil. In: ROUQUAYROL, M. Z.; ALMEIDA FILHO, N. Epidemiologia e Saúde. 6. ed. Rio de Janeiro: MEDSI; 2003. p. 587-603.

PAIM, J.S. Reforma Sanitária Brasileira: contribuição para a compreensão e crítica. / Salvador: EDUFBA; Rio de Janeiro: Ed. Fiocruz, 2008a.

PAIM, J. S. Reforma sanitária brasileira: eppur si muove. Caderno de Saúde Pública, Rio de Janeiro, v. 29, n. 10, p. 1927-1953, out. 2013 b.

PAIM, J. S. A reforma sanitária brasileira e o Sistema Único de Saúde: dialogando com hipóteses concorrentes. Physis, Rio de Janeiro, v. 18, n. 4, p. 625-644, 2008b.

PAIM, J. et. al. O sistema de saúde brasileiro: história, avanços e desafios. The Lancet, Estados Unidos da América, p. 12-31, 2011. Disponível em: <http://download.thelancet.com/flatcontentassets/pdfs/brazil/ brazilpor1.pdf $>$. Acesso em: 18 maio 2015.

PAIM, J. S.; TEIXEIRA, C. F. Política, planejamento e gestão em saúde: balanço do estado da arte. Revista Saúde Pública, Rio de Janeiro, n. 40, p. 73-78, 2006. Suplemento.

PAIVA, C. H. A.; TEIXEIRA, L. A. Reforma sanitária e a criação do Sistema Único de Saúde: notas sobre contextos e autores. História, Ciências, Saúde-Manguinhos, Rio de Janeiro, v. 21, n.1, p. 15-36, mar. 2014. PASCHE, D. F.; PASSOS, E.; HENNINGTON, É. A. Cinco anos da política nacional de humanização: trajetória de uma política pública. Ciência de Saúde Coletiva, Rio de Janeiro, v. 16, n. 11, p. 4541-4548, nov. 2011.

PELLEGRINI FILHO, A.; BUSS, P. M.; ESPERIDIÃO, M. A. Promoção da Saúde e seus fundamentos: determinantes sociais da saúde, ação intersetorial e políticas saudáveis. In: PAIM, J. S.; ALMEIDA FILHO, N. (Org.). Saúde coletiva: teoria e prática. Rio de Janeiro: Med Book. 2014. p. 305-326. 
PEPE, V. L. E. et al. A judicialização da saúde e os novos desafios da gestão da assistência farmacêutica. Ciência e Saúde Coletiva, Rio de Janeiro, v. 15, n. 5, p. 2405-2414, ago. 2010.

PIERANTONI, C. R. Formação de gestores para o SUS In: LEAL, M. C e FREITAS, C. M. (Org.). Cenários possíveis: experiências e desafios do mestrado profissional na saúde coletiva. Rio de Janeiro: Ed. Fiocruz, 2006. p. 123-136.

QUEIROZ, M. de S.; VIANNA, A. L. Padrão de política estatal em saúde e o sistema de assistência médica no Brasil atual. Revista de Saúde Pública, Rio de Janeiro, v. 26, n. 2, p. 132-140, abr. 1992.

(A) QUESTÃO democrática na área da saúde. Apresentado no $1^{\text {a }}$ Simpósio sobre Politica de Saúde da Câmara Federal,1979. Disponível em: <http://cebes.org.br/site/wp-content/uploads/2015/10/Cebes Saúde-e-Democracia.pdf $>$. Acesso: 4 out. 2015.

SAMPAIO, R. F.; MANCINI, M. C. Estudos de revisão sistemática: um guia para síntese criteriosa da evidência científica. Revista Brasileira de Fisioterapia, São Carlos, v. 11, n. 1, p. 83-89, jan./fev. 2007.

SANTOS NETO, E. T. dos. et al. Políticas de saúde materna no Brasil: os nexos com indicadores de saúde materno-infantil. Saúde e Sociedade, São Paulo, v. 17, n. 2, p. 107-119, Jun. 2008.

SANTOS, L. R. C. S.; SILVA, T. P. C. A utopia da Reforma Sanitária Brasileira em um discurso no Congresso da ABRASCO 2009. Saúde em debate, Rio de Janeiro, v. 37, n. 97, p. 210-2018, Jun. 2013.

SANTOS, N. R. dos. SUS, política pública de estado: seu desenvolvimento instituído e instituinte e a busca de saídas. Ciência de Saúde Coletiva, Rio de Janeiro, v. 18, n.1, p. 273-280, Jan. 2013.

SCHEFFER, M. O capital estrangeiro e a privatização do sistema de saúde brasileiro. Caderno de Saúde Pública, Rio de Janeiro, v. 31, n. 4, p. 663-666, abr. 2015.

SESTELO, J. A. de F.; SOUZA, L. E. P. F. de; BAHIA, L. Saúde suplementar no Brasil: abordagens sobre a articulação público/privada na assistência à saúde. Caderno Saúde Pública, Rio de Janeiro, v. 29, n. 5, p. 851-866, 2013. 
SOPHIA, D. C. Saúde e utopia: o Cebes e a reforma sanitária brasileira. São Paulo: Hucitec: Sobravime, 2015.

SOUZA, T. M. S. de; RONCALLI, A. G. Saúde bucal no Programa Saúde da Família: uma avaliação do modelo assistencial. Caderno de Saúde Pública, Rio de Janeiro, v. 23, n. 11, p. 2727-2739, nov. 2007.

TEIXEIRA, C. F.; PAIM, J. S. A política de saúde no governo Lula e a dialética do menos pior. Saúde em Debate, Rio de Janeiro, v. 29, n. 31, p. 268-283, 2005.

TEIXEIRA, C. F. Significado estratégico do Mestrado profissionalizante na consolidação do campo da saúde coletiva. In: LEAL, M. C.; FREITAS, C. M. (Org.). Cenários possíveis: experiências e desafios do mestrado profissional na saúde coletiva. Rio de Janeiro: Ed. Fiocruz, 2006. p. 33-47.

TEIXEIRA, C. F.; SOUZA, L. E.; PAIM, J. S. Sistema Único de Saúde, a difícil construção de um sistema universal na sociedade brasileira. In: ALMEIDA- FILHO, N.; PAIM, J. S. (Org.). Saúde coletiva: teoria e prática. Rio de Janeiro: Medbook, 2014. p. 121-137

TEIXEIRA, C. F. ; VILASBÔAS, A. L. Q. Modelos de atenção à saúde no SUS: transformação, mudança ou conservação. In: PAIM, J. S.; ALMEIDA-FILHO, N. de. Saúde coletiva: teoria e prática. Rio de Janeiro: MedBook, 2014. p. 287-301.

TEIXEIRA, C. F. Saúde da família, promoção e vigilância: construindo a integralidade da atenção à saúde no SUS. In: TEIXEIRA, C. F.; SOLLA, J. P. Modelo de atenção à saúde: vigilância e saúde da família. Salvador: EDUFBA, 2006. p. 59-83. (Sala de aula series, n. 3). Disponível em:<http://books.scielo.org>. Acesso em: 17 nov. 15.

TEIXEIRA, C. F. et al. Produção científica sobre política, planejamento e gestão no campo da saúde coletiva: visão panorâmica. In: PAIM, J. S.; ALMEIDA-FILHO, N. Saúde coletiva: teoria e prática. Rio de Janeiro: Medbook, 2014. p. 585-594.

TEIXEIRA, C. F.; SOLLA, J. P. Modelo de atenção à saúde: vigilância e saúde da família. Salvador: EDUFBA, 2006. (Sala de aula series, n. 3). Disponível em: <http://books.scielo.org>. Acesso em: 8 mar. 2013. 
TESTA, M. Pensamento estratégico e lógica de programação: o caso da saúde. São Paulo: Hucitec; Rio de Janeiro: ABRASCO, 1995.

VIEIRA, F. S. Assistência farmacêutica no sistema público de saúde no Brasil.Revista Panamericana de Salud Pública, Washington, v. 27, n. 2, feb. 2010.

VILLARINHO, M. V. et al. Políticas públicas de saúde face à epidemia da AIDS e a assistência às pessoas com a doença. Revista Brasileira de Enfermagem, Brasília, DF, v. 66, n. 2, p. 271-277, abr. 2013. 